\title{
Efficient Terahertz Emission from InGaN/GaN Heterostructure
}

\author{
A. REKLAitis \\ Semiconductor Physics Institute, Centre for Physical Sciences and Technology \\ A. Goštauto 11, LT-01108 Vilnius, Lithuania
}

\begin{abstract}
Terahertz emission from the freestanding InGaN/GaN heterostructure illuminated by femtosecond optical pulse is considered using Monte Carlo simulations. The results of Monte Carlo simulations show that the power of terahertz emission from InGaN/GaN heterostructure exceeds the power of the emission from InN surface by one order of magnitude.
\end{abstract}

PACS: 42.72.Ai, 73.20.Mf, 75.70.-i, 78.20.Bh, 78.47.-p

\section{Introduction}

Pulsed terahertz $(\mathrm{THz})$ radiation from semiconductors excited by femtosecond laser has been studied extensively in the recent years. The two main methods [1] are used to generate the broadband $\mathrm{THz}$ radiation. The methods are based on $\mathrm{THz}$ emission from the biased photoconductive switches and on $\mathrm{THz}$ emission from the surface of freestanding semiconductors. Multiple mechanisms are responsible for the $\mathrm{THz}$ emission from the freestanding semiconductor surface including nonlinear optical rectification, photocurrent induced by the surface-field, photocurrent induced by the built-in field in $p-i-n$ structures, and by photocurrent induced photo-Dember effect. However, the intensity of $\mathrm{THz}$ emission from the surface of freestanding semiconductors still is very low.

The analysis [2] of the transient dynamics of photoexcited carriers shows that the surface and contactless $p-i-n$ THz emitters have serious deficiencies that significantly reduce the intensity of $\mathrm{THz}$ radiation. First, only a minor part of the excited carriers contributes to the transient current if the inverse absorption coefficient exceeds the surface depletion width or the $i$-layer thickness of the $p-i-n$ structure. In this case, the major part of carriers is created in the region where the built-in electric field is screened by the extrinsic carriers. Second, the plasma frequency of photocarriers is position-dependent due to the exponential decay of the created carrier density. As a result, the transient response of the created carriers is incoherent in different regions of the structure because the frequency of current oscillations is position-dependent. Therefore, the amplitude of oscillations of the resulting current is significantly reduced by the interference effects.

To overcome such deficiencies of $\mathrm{THz}$ emitters, the novel $\delta$-doped GaAs/AlGaAs heterostructure is suggested in [2]. It is shown in [2] that $\mathrm{THz}$ energy radiated from the freestanding semiconductors is significantly enhanced when the above noted deficiencies are eliminated. It is obtained from Monte Carlo simulations that the efficiency of $\mathrm{THz}$ emission from the $\delta$-doped GaAs/AlGaAs heterostructure exceeds the efficiency of $\mathrm{THz}$ emission from the homogeneous GaAs by two orders of magnitude.

In the present work, the basic ideas suggested in [2] are used to design $\mathrm{THz}$ emitters based on InGaN/GaN heterostructures. The pulsed $\mathrm{THz}$ emission from the InGaN/GaN heterostructures is studied using Monte Carlo simulations [2, 3] of the transient dynamics of photoexcited electron-hole plasma. The InGaN/GaN heterostructures appear to be very suitable for the development of highly efficient $\mathrm{THz}$ emitters. High electron mobility in the optically active InGaN layers provides a large amplitude of the transient current. The offsets of the conduction and valence bands at the $\mathrm{InGaN} / \mathrm{GaN}$ heterointerfaces are sufficiently high to ensure the perfect confinement of the excited carriers inside the InGaN layers. In addition to the basic ideas of [2], the polarization charges at the InGaN/GaN interfaces are employed in the design of the appropriate potential profile in the InGaN/ GaN heterostructures for the efficient $\mathrm{THz}$ emission.

\section{Model of InGaN/GaN heterostructure}

The parameters of the conduction band and electron scattering for InGaN/GaN heterostructures are taken from the set of parameters listed in [4] for $\mathrm{InN} / \mathrm{GaN}$ heterostructure. The linear interpolation between $\mathrm{InN}$ and GaN is used for the evaluation of parameters for $\mathrm{In}_{1-x} \mathrm{Ga}_{x} \mathrm{~N} / \mathrm{GaN}$ heterostructures. The material parameters for valence band of $\mathrm{InGaN} / \mathrm{GaN}$ heterostructures are taken from [5]. The surface Fermi level pinned at $1.6 \mathrm{eV}$ above the valence band maximum $[6,7]$ is accepted in the simulations.

The simulations of the optically excited electron-hole plasma are carried out by ensemble Monte Carlo method as described in $[2,3]$. The simulations are started from the thermally equilibrium distribution of carriers. The duration of the optical pulse $\tau_{\mathrm{F}}$ is set to $100 \mathrm{fs}$. The performance of the InGaN/GaN $\mathrm{THz}$ emitter is studied for the $800 \mathrm{~nm}$ wavelength of the optical excitation. The absorption coefficients of the $\mathrm{In}_{1-x} \mathrm{Ga}_{x} \mathrm{~N}$ layers for this wavelength are evaluated from the absorp- 
tion spectrum of InN [8] using the linear extrapolation to the $\mathrm{In}_{1-x} \mathrm{Ga}_{x} \mathrm{~N}$ band-gap. The estimated absorption coefficient of $\operatorname{In}_{1-x} \mathrm{Ga}_{x} \mathrm{~N}$ ranges between $7 \times 10^{4} \mathrm{~cm}^{-1}$ and $2 \times 10^{4} \mathrm{~cm}^{-1}$ for $x$ ranging between $x=0$ and $x=0.28$, respectively. The absorption coefficient $\alpha=$ $2.7 \times 10^{4} \mathrm{~cm}^{-1}$ is taken for $\operatorname{In}_{0.75} \mathrm{Ga}_{0.25} \mathrm{~N}$.

Several structures of the $\mathrm{In}_{1-x} \mathrm{Ga}_{x} \mathrm{~N} / \mathrm{GaN} \mathrm{THz}$ emitter have been investigated for the best performance. The optimized structure is composed from four periods of narrow band-gap optically-active $n$ - $\operatorname{In}_{0.75} \mathrm{Ga}_{0.25} \mathrm{~N}$ layers doped to $10^{17} \mathrm{~cm}^{-3}$ and from wide band-gap $n$-GaN barriers doped to $2 \times 10^{17} \mathrm{~cm}^{-3}$. The thickness $L_{i}$ of each InGaN layer is taken as $110 \mathrm{~nm}$. The thickness of GaN barriers is taken as $20 \mathrm{~nm}$. The simulated structure is completed by the substrate layer of $150 \mathrm{~nm}$ doped to $10^{17} \mathrm{~cm}^{-3} n-\operatorname{In}_{0.75} \mathrm{Ga}_{0.25} \mathrm{~N}$. The optimized structure is shown in Table.

TABLE

Schematic diagram of the $\operatorname{In}_{1-x} \mathrm{Ga}_{x} \mathrm{~N} / \mathrm{GaN} \mathrm{THz}$ emitter.

\begin{tabular}{c|c|c}
\hline \hline Layer & Thickness $[\mathrm{nm}]$ & Doping $\left[\mathrm{cm}^{-3}\right]$ \\
\hline$n$-In $0.75 \mathrm{Ga}_{0.25} \mathrm{~N}$ & 110 & $1 \times 10^{17}$ \\
$n$-GaN barrier & 20 & $2 \times 10^{17}$ \\
$n$-In $0.75 \mathrm{Ga}_{0.25} \mathrm{~N}$ & 110 & $1 \times 10^{17}$ \\
$n$-GaN barrier & 20 & $2 \times 10^{17}$ \\
$n$-In ${ }_{0.75} \mathrm{Ga}_{0.25} \mathrm{~N}$ & 110 & $1 \times 10^{17}$ \\
$n$-GaN barrier & 20 & $2 \times 10^{17}$ \\
$n$-InN & 110 & $1 \times 10^{17}$ \\
$n$-GaN barrier & 20 & $2 \times 10^{17}$ \\
$n$-In 0.75 Ga0.25N & 150 & $1 \times 10^{17}$ \\
$($ substrate) & &
\end{tabular}

\section{Results and discussion}

The calculated equilibrium potential profile and carrier distribution in the four-period $\mathrm{InGaN} / \mathrm{GaN}$ emitter are presented in Fig. 1. The polarization charges at InGaN/ GaN interfaces provide a high built-in electric field in the optically active InGaN layers. The average built-in electric field in the InGaN layers is close to $\varepsilon_{\mathrm{g}} / e L_{i}$, where $\varepsilon_{\mathrm{g}}$ is the band-gap energy and $e$ is the electron charge. The strength of the average built-in electric field reaches $110 \mathrm{kV} / \mathrm{cm}$ in the InGaN layers. The high built-in electric field ensures a fast acceleration of photoexcited carriers, and thus, high amplitude of transient photocurrent.

The comparison of the transient photocurrent in $n$-InN and $n$-InGaN/GaN heterostructure after the optical pulse duration of $100 \mathrm{fs}$ is shown in Fig. 2. The transient photocurrent in homogeneous $\mathrm{InN}$ is induced by the photo-Dember effect due to downward band bending at InN surface [9]. Contrary to that, the photocurrent in $\mathrm{InGaN} / \mathrm{GaN}$ heterostructure is induced by the strong polarization charges at the $\mathrm{InGaN} / \mathrm{GaN}$ heterointerfaces, and the photo-Dember effect is completely suppressed. Therefore, the transient photocurrents in InN

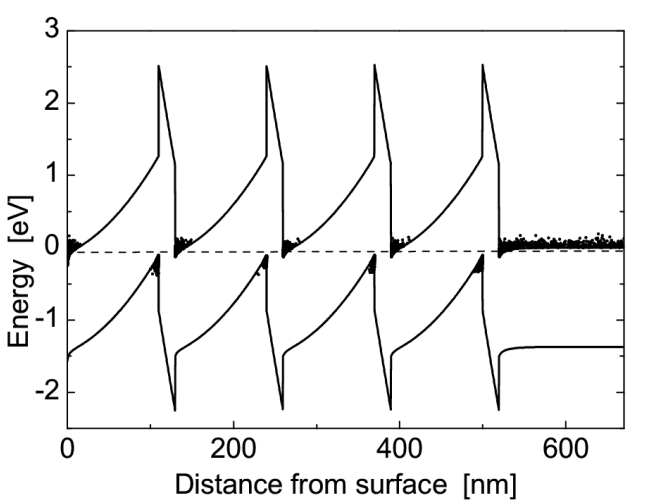

Fig. 1. Equilibrium potential profile (conduction and valence band edges) in the InGaN/GaN emitter (solid curves) and electron and hole kinetic energies (points) before photoexcitation. The dashed line shows the Fermi level.

and InGaN/GaN are of opposite directions. The homogeneous InN is identified as one of the most efficient emitters of pulsed $\mathrm{THz}$ radiation [9]. The results presented in Fig. 2 show that the efficiency of the heterostructure InGaN/GaN emitter essentially exceeds the efficiency of the InN emitter. The amplitude of the transient photocurrent in the InGaN/GaN heterostructure is approximately five times higher than the transient photocurrent in the $\mathrm{InN}$.

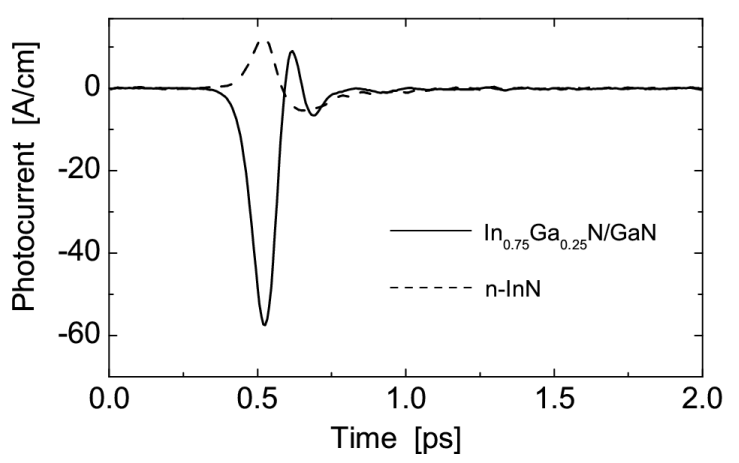

Fig. 2. Waveforms of the transient current in the InGaN/GaN emitter (solid curve) and in the $n-\mathrm{InN}$ doped to $10^{17} \mathrm{~cm}^{-3}$ (dashed curve). The optical fluence is $10 \mu \mathrm{J} / \mathrm{cm}^{2}$. The peak of the optical pulse intensity is at $t=0.5 \mathrm{ps}$, and $\tau_{\mathrm{F}}$ is set to $100 \mathrm{fs}$.

The energy and spectrum of $\mathrm{THz}$ radiation are obtained from the simulated transient current of photoexcited carriers. The time-dependent $\mathrm{THz}$ power $P(t)$ radiated in all directions by the electric dipole moment $\boldsymbol{D}(t)$ and the first derivative of the dipole moment are evaluated as [2]:

$$
P(t)=\frac{1}{6 \pi \varepsilon c^{3}}\left(\frac{\mathrm{d}^{2} \boldsymbol{D}(t)}{\mathrm{d} t^{2}}\right)^{2}
$$

and 


$$
\frac{\mathrm{d} \boldsymbol{D}(t)}{\mathrm{d} t}=\int \boldsymbol{j}(\boldsymbol{r}, t) \mathrm{d} V,
$$

where $\varepsilon$ is the vacuum permittivity, $c$ is the speed of light, $\boldsymbol{j}(\boldsymbol{r}, t)$ is the current density of electrons and holes, and the integration is taken over the volume $V$ of the photoexcitation. The total $\mathrm{THz}$ energy radiated in all directions is obtained by the integration of $P(t)$ over the duration of transient evolution of photoexcited carriers. The spectrum of radiation is found from the Fourier analysis of $P(t)$. The calculated spectra of radiation are shown in Fig. 3.

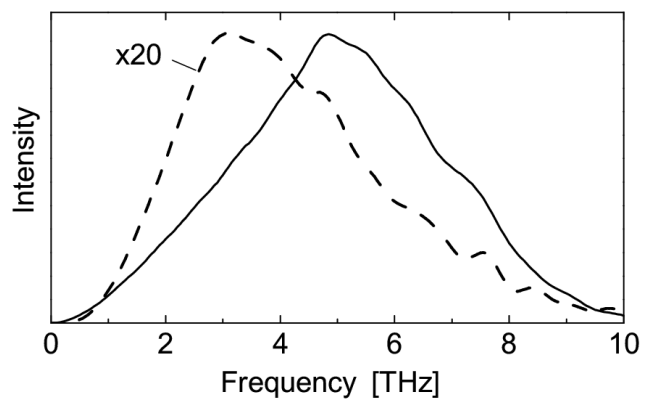

Fig. 3. Spectra of $\mathrm{THz}$ emission from $\mathrm{InGaN} / \mathrm{GaN}$ emitter (solid curve) and from $n$-InN (dashed curve). The optical fluence is $10 \mu \mathrm{J} / \mathrm{cm}^{2}$, and $\tau_{\mathrm{F}}$ is $100 \mathrm{fs}$.

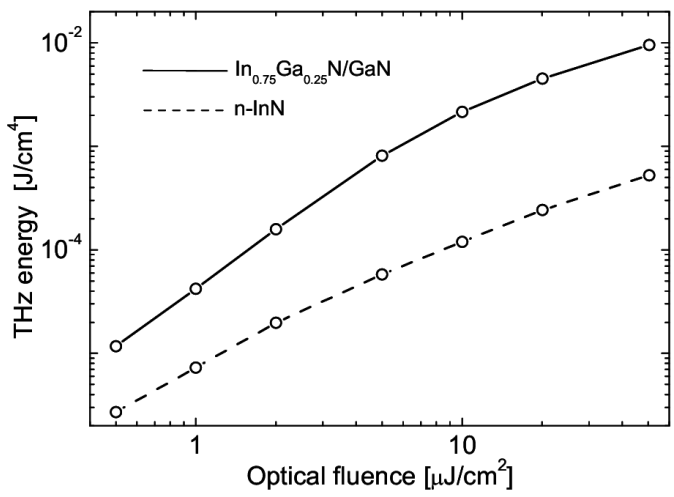

Fig. 4. Radiated $\mathrm{THz}$ energy as a function of the optical fluence. The solid and dashed curves present the energy radiated from the four-period $\mathrm{InGaN} / \mathrm{GaN}$ heterostructure and from $n$-InN doped to $10^{17} \mathrm{~cm}^{-3}$, respectively. The pulse duration $\tau_{\mathrm{F}}$ is set to $100 \mathrm{fs}$.

The comparison of the intensity of $\mathrm{THz}$ emission from the homogeneous $n$-InN and from the InGaN/GaN heterostructure is presented in Fig. 4. Due to downward bend bending, $\mathrm{THz}$ emission from the $\mathrm{InN}$ is caused by the relatively weak photo-Dember effect [9]. Contrary to that, $\mathrm{THz}$ emission from the $\mathrm{In}_{1-x} \mathrm{Ga}_{x} \mathrm{~N} / \mathrm{GaN}$ heterostructure is induced by the strong built-in electric field. Therefore, the intensity of $\mathrm{THz}$ emission from the $\mathrm{In}_{1-x} \mathrm{Ga}_{x} \mathrm{~N} / \mathrm{GaN}$ heterostructure essentially exceeds the intensity of $\mathrm{THz}$ emission from the $\mathrm{InN}$ surface. The dependence of intensity of $\mathrm{THz}$ emission from the $\mathrm{In}_{1-x} \mathrm{Ga}_{x} \mathrm{~N} / \mathrm{GaN}$ heterostructure on the optical fluence $I_{0}$ is near-proportional to $I_{0}^{2}$ in the range $I_{0} \leq 10 \mu \mathrm{J} / \mathrm{cm}^{2}$.
According to the hydrodynamic theory [2] of heterostructure emitters, the emitted $\mathrm{THz}$ energy is proportional to the squared built-in electric field and inversely proportional to the momentum relaxation rate $\gamma$. The momentum relaxation rate in $\mathrm{InN}$ is evaluated using Monte Carlo method suggested in [10]. It is found that the momentum relaxation rate of photoelectrons in $\mathrm{In}_{0.75} \mathrm{Ga}_{0.25} \mathrm{~N}$ excited by $800 \mathrm{~nm}$ optical pulse is $6 \times 10^{12} \mathrm{~s}^{-1}$ which is close to momentum relaxation rate $7 \times 10^{12} \mathrm{~s}^{-1}$ in GaAs [2]. Therefore, one could expect that the efficiency of the InGaN/GaN heterostructure emitter should be of the same order as the GaAs/AlGaAs emitter considered in [2]. The results of Monte Carlo simulations of InGaN/GaN and GaAs/AlGaAs THz emitters confirm this expectation. Moreover, the InGaN/GaN heterostructures have a significant advantage in the development of efficient $\mathrm{THz}$ emitters because the role of $\delta$-doped layers in GaAs/AlGaAs emitter is played by the inherent polarization charges in $\mathrm{InGaN} / \mathrm{GaN}$ emitter.

\section{Conclusions}

In conclusion, the efficient InGaN/GaN emitter of pulsed $\mathrm{THz}$ radiation is proposed and investigated using Monte Carlo simulations. The THz energy emitted from the suggested InGaN/GaN emitter exceeds the energy of $\mathrm{THz}$ radiation from $\mathrm{InN}$ by one order of magnitude.

\section{Acknowledgments}

This work was supported by the Research Council of Lithuania under contract No. MIP-49/2010.

\section{References}

[1] Terahertz Optoelectronics, Ed. K. Sakai, Springer, Berlin 2005.

[2] A. Reklaitis, Phys. Rev. B 77, 153309 (2008).

[3] A. Reklaitis, Phys. Rev. B 74, 165305 (2006).

[4] A. Reklaitis, J. Phys., Condens. Matter 20, 384202 (2008).

[5] I. Vurgaftman, J.R. Meyer, J. Appl. Phys. 94, 3675 (2003).

[6] I. Mahboob, T.D. Veal, C.F. McConville, H. Lu, W.J. Schaff, Phys. Rev. Lett. 92, 036804 (2004).

[7] I. Mahboob, T.D. Veal, L.F.J. Pipper, C.F. McConville, H. Lu, W.J. Schaff, J. Furthmüller, F. Bechstedt, Phys. Rev. B 69, R201307 (2004).

[8] J. Wu, W. Walukiewicz, S.X. Li, R. Armitage, J.C. Ho, E.R. Weber, E.E. Haller, H. Lu, W.J. Schaff, A. Barcz, R. Jakiela, Appl. Phys. Lett. 84, 2805 (2004).

[9] R. Ascazubi, I. Wilke, K. Denniston, H. Lu, W.J. Schaff, Appl. Phys. Lett. 84, 4810 (2004).

[10] Yu. Pozhela, A. Reklaitis, Fiz. Tekh. Poluprovodn. (S.-Petersburg) 13, 1127 (1979) [Sov. Phys.-Semicond. 13, 660 (1979)]. 